\title{
Online-Offline Optimized Motion Profile for High-Dynamic Positioning of Ultraprecision Dual Stage
}

\author{
Yang Liu $\mathbb{D}^{1,2}$ Yue Dong $\mathbb{D}^{1,2}$ and Jiubin $\operatorname{Tan}^{2}$ \\ ${ }^{1}$ Department of Control Science and Engineering, Harbin Institute of Technology, Harbin 150001, China \\ ${ }^{2}$ Center of Ultra-Precision Optoelectronic Instrument, Harbin Institute of Technology, Harbin 150001, China \\ Correspondence should be addressed to Yue Dong; yuedong4206@gmail.com
}

Received 14 April 2018; Revised 3 July 2018; Accepted 18 July 2018; Published 23 August 2018

Academic Editor: Yimin Zhou

Copyright ( 2018 Yang Liu et al. This is an open access article distributed under the Creative Commons Attribution License, which permits unrestricted use, distribution, and reproduction in any medium, provided the original work is properly cited.

\begin{abstract}
The wafer stage in dual-stage lithographic system is an air-bearing servo motion platform requiring high positioning accuracy and high transient performance. However, the residual vibration, resulting from almost zero damping, high velocity, parallel decoupling structure, and direct drive, brings about unacceptable overshoot and settling time. To suppress these unfavorable elements in high dynamic motion, a novel motion profile planning method combined with input shaping is proposed in this paper. Firstly, a trajectory named all free S-curve (AFS-curve) is derived, which has less constraints and better performance than traditional S-curve profile. Then, AFS-curve combined with a zero vibration shaper (ZV) is developed to further suppress residual vibration. Due to the very complex parameter adjustment, the online tuning may cause system oscillation that leads to damage of the precision stage. This paper, furthermore, proposes an online-offline method to optimize the parameters in the motion profile. Online step is performed to collect input and output data. Offline step includes the system model identification based on I/O data and parameter self-learning based on particle swarm optimization (PSO). The simulation and experimental results indicate that the proposed method achieves significant reduction of the positioning time and the overshoot in the dual-stage system.
\end{abstract}

\section{Introduction}

The wafer stage of lithography is a high-speed and highaccuracy motion platform [1], which is used for bearing wafers. To improve throughput of lithography, there are always two wafer stages constituting the dual-stage system [2]. The two wafer stages work at the same time, one for preprocessing and the other for exposure. From this working mode, we know that when exchanging two wafer stages, there is no wafer to be exposed. Thus, reducing the exchanging time is crucial to the improvement of the lithographic throughput. However, the mechanical structure of the system is fixed, so the exchanging path is constant. Therefore, shorter exchanging time means larger average velocity. Meanwhile, the aerostatic guide supporting the platform leads to light damping. As a result, the residual vibrations are easily introduced in wafer stage systems. Considering the above adverse factors and the system requirements comprehensively, the trajectory planning method is widely concerned. Motion profile is a technique that can reduce the residual vibration, and the trapezoidal velocity curve is the most primitive type $[3,4]$. The trapezoidal velocity curve involves infinite jerk that can describe the changes of acceleration, which is usually positively related with the residual vibration. Hence, jerk value should be limited. Compared to the trapezoidal curve, S-curve constrains the jerk to a constant value, which is more widely used for motion profile due to less residual vibration [5-12]. Traditional symmetric S-curve profiles have been widely adopted in many motion control fields [7-9]. The generating methods of symmetric S-curve include finite-impulse response filtering technique [10] and optimal control approach [11, 12]. However, there are still constraints in generating traditional S-curve, for example, the jerk at acceleration period should have equal value, resulting in limited performance. Thus, this paper presents a method to generate the S-curve profile named AFS-curve, which could relax the constraint maximally.

As long as the motion profile and natural frequency of the system are known exactly, the input-shaping method (IS) could be very effective to suppress the residual vibration 


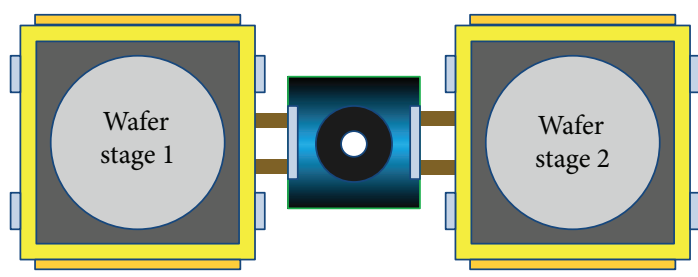

Figure 1: The sketch diagram of dual stage.

and the overshoot [13-15]. Meanwhile, as a prefilter, the S-curve could also be beneficial to the vibration abatement. To achieve desired performance, the motion profile should be shaped by input shaper. Input shaper [16], one kind of feedforward controllers, shapes the reference input signal based on the characteristics of the controlled system. It consists of two or more groups of signals with different amplitudes and delay times. These signals result in different responses, the superposition of which could reduce the oscillation amplitude, even to zero. References [17-19] proposed concepts of zero-vibration ( $\mathrm{ZV})$, zero-vibration-derivative (ZVD), and extra insensitive (EI) shapers. Furthermore, to enhance the robustness against parameter uncertainty, the $\mathrm{ZVD}^{\mathrm{k}}$ input shaper was proposed, but produced a longer time delay with smaller step size. As the settling time is the major concern during the swap of the wafer stages, the ZV shaper has been selected in this paper.

As mentioned above, both of the AFS-curve and ZV shaper have at least three parameters, which depend on the accurate model of the controlled system; however, it is a tough task to build the model of a highly coupled system. Thus, a reliable optimization algorithm [20-23] is more suitable for the trajectory planning. Furthermore, both of the overshoot and error should be considered in the optimization object, so a multiobjective optimization based on PSO [24-29] (particle swarm optimization) is adopted in this paper.

Subsequently, if the AFS-curve and input shaper parameters are optimized online, it is easy to cause a serious system shock, even damage some components. Therefore, the parameter optimization phase should be carried out offline. Finally, an online-offline method to optimized motion profile is proposed in this paper to suppress the residual vibration of rotary motor and reduce the positioning time of the dual stage.

\section{Problem Formulation}

In order to increase the wafer throughput, the dual-stage structure is adopted in lithographic machine, as seen in Figure 1, whose working region is composed by preprocessing part and exposure part. In preprocessing region, the wafer stage conducts leveling, focusing, and measuring the surface topography of the wafer. The exposure operation is carried out simultaneously in the exposure region. As a result, there is no need to adjust the wafer before exposure, just exchanging two wafer stages, saving much time than conventional single-stage lithography equipment. To implement the exchanging operation, three rotary electric motors are involved, one named common revolution motor and the others named private rotation motors. The communal motor is used to exchange the positions of stage 1 and stage 2 , and the two private motors are designed for adjusting stages during rotation, avoiding cable twining. In this paper, we focus on the control problems of communal motor that occur in exchanging period. In the other words, for light damping and heavy load, the performance of overshoot and settling time should be considered.

For the synchronization of the three motors, different disturbances should be dispelled at the same time, which include thrust ripple, frictional force, cable force, and interaction force caused by asynchronous motion. These disturbances are relevant to different physical quantities. For instance, thrust ripple is related with position, and it can be calculated by a rough mechanism model, but the tracking error from modeling indeterminacy may be increased for the synchronized motion. Frictional forces from cable disturbing and relative motion between the stator and mover are related with velocity, and it can be obtained by the formula of the friction force; however, different positions have different friction coefficients for tooling problems, that is to say, it is difficult to obtain a series of accurate friction coefficients. Similarly, different speeds lead to different centrifugal forces, which signify different frictional forces. Interaction force is related with acceleration and deceleration, and it should be pointed out that the controlled system is highspeed high-precision equipment, which indicates that acceleration and deceleration always exceed the rated values that the motors can provide. As a result, the motors may work under nonlinearity when the system requires high acceleration or deceleration. Meanwhile, different motors have different saturation nonlinearity characters; thus, the output of different motors may not be completely synchronized, leading to an interaction force between communal motor and the private motor. In a word, these disturbances make it hard to obtain the accurate model of the dual stage based on mechanism.

Although these disturbances are not easy to be measured and modeled, most of them are repeatable based on the same tracking trajectory, and the repeatable disturbances are the main factors that influence the system dynamic performance. Therefore, a black box method, which is a high-order linear system model identification based on input/output data, is proposed to build the mathematical model of the system. The implementation of this method is based on MATLAB system identification toolbox. In detail, firstly, define the upper limit $n_{\max }$ to be the highest order of model's numerator and denominator. Thus, the dual-stage system model can be expressed as follows:

$$
G(s)=\frac{a_{n} s^{n}+a_{n-1} s^{n-1}+\cdots+a_{1} s+a_{0}}{b_{m} s^{m}+b_{m-1} s^{m-1}+\cdots+b_{1} s+b_{0}},
$$

where $n \leqq n_{\max }, m \leqq n_{\max }$ and $a_{0}, a_{1}, \ldots, a_{n}$ and $b_{0}, b_{1}, \ldots, b_{m}$ are the coefficients of numerator and denominator. Then, reduce the highest order of model's numerator and denominator; meanwhile, make use of the actual sampling data to identify the system model corresponding to every order 


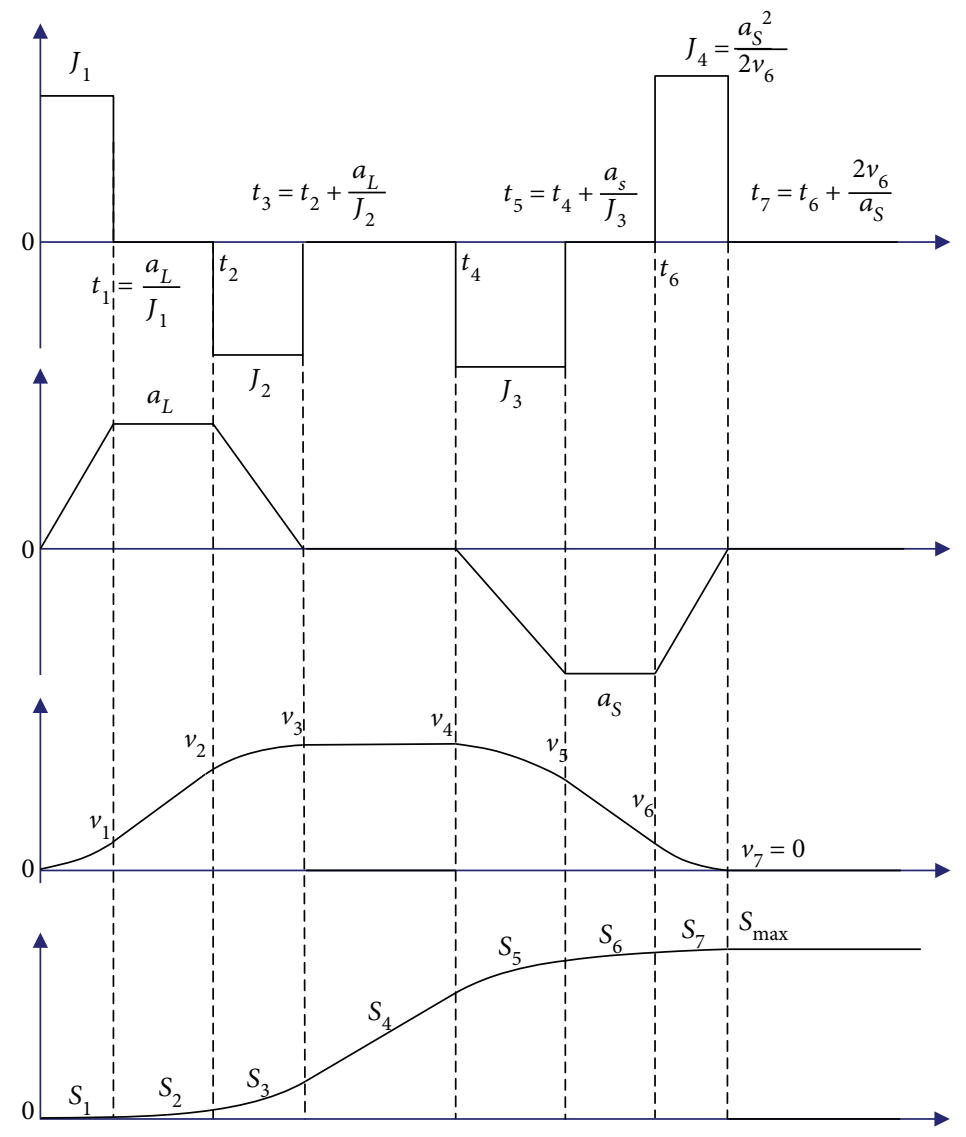

FIgURE 2: S-curve motion profiles and notations.

change, until $n=0$ and $m=0$. Comparing the modeling errors, this paper selects the most suitable system model according to the least mean square error principle.

\section{AFS-Curve Combined Input Shaping}

The aforementioned disturbances are relevant to the actual position, speed, and acceleration of the stage. Once a closed-loop system is determined, a better motion profile that suits the controlled system could suppress the disturbance and residual vibration to achieve a better dynamic performance. Usually, trajectory planning and input shaping technique are the two most popular methods to get a desired motion profile. It should be pointed out that the S-type trajectory is more widely used in reference trajectory planning than the trapezoidal curve. For its less residual vibration characteristic, zero vibration input shaping is widely applied in many practical applications. Hence, we will emphatically discuss how to formulate an S-curve in conventional and all free ways, then propose a new method for all free S-curve combined with input shaping (AFS-IS). Two basis conditions of the S-type trajectory compared in this paper should be assured by the following constraints. Firstly, position should start at 0 and end at $S_{\max }$. Secondly, velocity, acceleration, and jerk at the starting and ending points must be zero. Figure 2 shows S-curve motion profiles and notations, with the absolute values of variables.
3.1. Conventional S-Curve. The acceleration, deceleration, and velocity of conventional S-curve are all symmetric, which means the four parameters are equaled, which could be expressed as follows:

$$
\begin{aligned}
J_{1} & =J_{2}=J_{3}=J_{4}=J, \\
a_{L} & =a_{S}=A, \\
t_{2}-t_{1} & =t_{6}-t_{5}=t_{a} .
\end{aligned}
$$

Then, the function of time and position can be derived.

$$
\begin{aligned}
s_{1}= & \frac{J t^{3}}{6}, \\
s_{2}= & \frac{a t^{2}}{2}-\frac{a^{2} t}{2 J}+\frac{a^{3}}{6 J^{2}}, \\
s_{3}= & -\frac{J t^{3}}{6}+\left(a+\frac{J t_{a}}{2}\right) t^{2}-\left(\frac{a^{2}}{J}+a t_{a}+\frac{J t_{a}^{2}}{2}\right) t+\frac{J t_{a}^{3}}{6}+\frac{a t_{a}^{2}}{2} \\
& +\frac{a^{2} t_{a}}{2 J}+\frac{a^{3}}{3 J^{2}}, \\
s_{4}= & \left(t_{a}+\frac{a}{J}\right) a t-\frac{a}{2}\left(t_{a}^{2}+\frac{3 a t_{a}}{J}+\frac{2 a^{2}}{J^{2}}\right), \\
s_{5}= & -\frac{J t^{3}}{6}+\frac{J t^{2}}{2}\left(t_{v}+\frac{2 a}{J}+t_{a}\right)
\end{aligned}
$$




$$
\begin{aligned}
& -\left(\frac{J t_{v}^{2}}{2}+2 a t_{v}+J t_{a} t_{v}+\frac{a^{2}}{J}+a t_{a}+\frac{J t_{a}^{2}}{2}\right)-\frac{a}{2} t\left(\frac{a}{J}+t_{a}\right)^{2} \\
& -\frac{a^{2}}{2 J}\left(\frac{a}{J}+t_{a}\right)-\frac{J}{6}\left(t_{v}+\frac{2 a}{J}+t_{a}\right)^{3}, \\
s_{6}= & -\frac{a t^{2}}{2}+\left(2 a t_{a}+a t_{v}+\frac{7 a^{2}}{2 J}\right) t-\frac{a t_{a}}{2}\left(\frac{a}{J}+t_{a}\right) \\
& -\frac{a}{2}\left(t_{v}+\frac{2 a}{J}+t_{a}\right)^{2}-\frac{a^{2}}{2 J}\left(t_{v}+\frac{2 a}{J}+t_{a}\right)-\frac{a^{3}}{6 J^{2}}, \\
s_{7} & =\frac{J t^{3}}{6}-\left(2 a+J t_{a}+\frac{J t_{v}}{2}\right) t^{2}+\left(\frac{J t_{6}^{2}}{2}+\frac{a^{2}}{2 J}+a t_{6}\right) t-\frac{J t_{6}^{3}}{6} \\
& -\frac{a t_{4}^{3}}{2}-\frac{a^{2} t_{4}}{2 J}-\frac{a t_{2}^{2}}{2}-\frac{a^{2} t_{2}}{2 J}-\frac{a^{3}}{6 J^{2}},
\end{aligned}
$$

where $t_{2}=a /\left(J+t_{a}\right), t_{4}=\left(t_{v}+2 a\right) /\left(J+t_{a}\right)$.

\subsection{All Free S-Curve}

3.2.1. Formulating All Free S-Curve. All free S-curve (AFS-curve) is a novel method to describe S-curve. AFS-curve does not constrain any variable, which is used for generating the motion profile. That is to say, $J_{1}, a_{L}, t_{2}, J_{2}, t_{4}$, $J_{3}$, and $a_{S}$ are independent to each other. Thus, AFS-curve can represent all the characteristic of S-curve with $J_{1}, a_{L}, t_{2}$, $J_{2}, t_{4}, J_{3}$, and $a_{S}$. The time position function of AFS-curve can be expressed by

$$
\begin{aligned}
s_{1}= & \frac{J_{1} t^{3}}{6}, \\
s_{2}= & \frac{a_{L} t^{2}}{2}-\frac{a_{L}^{2} t}{2 J_{1}}+\frac{a_{L}^{3}}{6 J_{1}^{2}}, \\
s_{3}= & -\frac{J_{2} t^{3}}{6}+\frac{\left(a_{L}+J_{2} t_{2}\right) t^{2}}{2}-\frac{\left(\left(J_{2} t_{2}^{2}+a_{L}^{2}\right) / J_{1}\right) t}{2}+\frac{J_{2} t_{2}^{3}}{6}+\frac{a_{L}^{3}}{6 J_{1}^{2}}, \\
s_{4}= & \left(\frac{a_{L}^{2}}{2 J_{2}}+a_{L} t_{2}-\frac{a_{L}^{2}}{2 J_{1}}\right) t-\frac{a_{2} t_{2}^{2}}{2}-\frac{a_{L}^{2} t_{2}}{2 J_{2}}-\frac{a_{L}^{3}}{6 J_{2}^{2}}+\frac{a_{L}^{3}}{6 J_{1}^{2}}, \\
s_{5}= & -\frac{J_{3} t^{3}}{6}+\frac{J_{3} t_{4} t^{2}}{2}+\left(\frac{a_{L}^{2}}{2 J_{2}}+a_{L} t_{2}-\frac{a_{L}^{2}}{2 J_{1}}-\frac{J_{3} t_{4}^{2}}{2}\right) t+\frac{J_{3} t_{4}^{3}}{6} \\
& -\frac{a_{L}^{2} t_{2}^{2}}{2}-\frac{a_{L}^{2} t_{2}}{2 J_{2}}-\frac{a_{L}^{3}}{6 J_{2}^{2}}+\frac{a_{L}^{3}}{6 J_{1}^{2}}, \\
s_{6}= & -\frac{a_{s} t^{2}}{2}+\left(\frac{a_{L}^{2}}{2 J_{2}}+a_{L} t_{2}-\frac{a_{L}^{2}}{2 J_{1}}+\frac{a_{s}^{2}}{2 J_{3}}+a_{s} t_{4}\right) t-\frac{a_{s} t_{4}^{2}}{2} \\
& -\frac{a_{L} t_{2}^{2}}{2}-\frac{a_{L}^{2} t_{2}}{2 J_{2}}-\frac{a_{L}^{3}}{6 J_{2}^{2}}+\frac{a_{L}^{3}}{6 J_{1}^{2}}-\frac{a_{s}^{3}}{6 J_{3}{ }^{2}}-\frac{a_{s}^{2} t_{4}}{2 J_{3}}, \\
s_{7} & =\frac{a_{s}^{2} t^{3}}{12 v_{6}}-\frac{\left(\left(a_{s}+a_{s}^{2} t_{6}\right) / 2 v_{6}\right) t^{2}}{2}+\left(a_{s} t_{6}+\frac{a_{s}^{2} t_{6}^{2}}{4 v_{6}}+v_{6}\right) t-\frac{a_{s} t_{6}^{2}}{2} \\
& -\frac{a_{s}^{2} t_{6}^{3}}{12 v_{6}}-v_{6} t_{6}+s_{6} .
\end{aligned}
$$

The computational process of $t_{6}$ will be given later.
3.2.2. Constraints of AFS-Curve. In AFS-curve, the time points increases from $t_{1}$ to $t_{7}$; however, $t_{1}, t_{3}$, and $t_{5}$ are expressed by forward free variables. Thus, $t_{2}>t_{1}=a_{L} / J_{1}$, $t_{4}>t_{3}=t_{2}+a_{L} / J_{2}$, and $t_{6}>t_{5}=t_{4}+a_{S} / J_{3}$. To facilitate different application environment, a few conditions should be clarified.

(1) The maximum shock is limited, then it follows that

$$
J_{1} \leq J_{\max }, J_{2} \leq J_{\max }, J_{3} \leq J_{\max }, J_{4} \leq J_{\max },
$$

Because $J_{4}=a_{S}{ }^{2} /\left(2 v_{6}\right) \leq J_{\max }$, where $v_{6}=\left(-a_{S} t_{6}+\right.$ $\left.a_{L}^{2}\right) /\left(2 J_{2}\right)+\left(a_{L} t_{2}-a_{L}^{2}\right) /\left(2 J_{1}\right)+a_{S}^{2} /\left(2 J_{3}\right)+a_{S} t_{4}$ is the velocity at time $t_{6}$, so the following is derived.

$$
t_{6}<-\frac{a_{s}}{2 J_{\max }}+\frac{a_{s}}{2 J_{3}}+\frac{a_{L}^{2}}{2 J_{2} a_{s}}-\frac{a_{L}^{2}}{2 J_{1} a_{s}}+\frac{a_{L} t_{2}}{a_{s}}+t_{4} .
$$

(2) The maximum force is limited, then it follows that

$$
a_{L}=\left(t_{3}-t_{2}\right) J_{2} \leq a_{\max }, a_{s}=\left(t_{5}-t_{4}\right) J_{3} \leq a_{\max } .
$$

Thus,

$$
t_{3} \leq \frac{a_{\max }}{J_{3}}+t_{2}, t_{5} \leq \frac{a_{\max }}{J_{3}}+t_{4} .
$$

(3) The maximum centrifugal force or friction force is limited, then it follows that

$$
v_{3}=v_{4} \leq v_{\max } .
$$

Thus,

$$
J_{2} \geq \frac{a_{L}^{2}}{2\left(v_{\max }+\left(a_{L}^{2} / 2 J_{1}\right)-a_{L} t_{2}\right)} .
$$

(4) The maximum distance is fixed, then it follows that

$$
S_{7}=S_{6}+\frac{2 v_{6}^{2}}{3 a_{S}}=S_{\max }
$$

where $S_{6}$ is the position at time point $t_{6}$, following that

$$
\begin{aligned}
S_{6}= & -\frac{a_{s} t_{6}^{2}}{2}+\left(\frac{a_{L}^{2}}{2 J_{2}}+a_{L} t_{2}-\frac{a_{L}^{2}}{2 J_{1}}+\frac{a_{s}^{2}}{2 J_{3}}+a_{s} t_{4}\right) t_{6}-\frac{a_{s} t_{4}^{2}}{2} \\
& -\frac{a_{L} t_{2}^{2}}{2}-\frac{a_{L}^{2} t_{2}}{2 J_{2}}-\frac{a_{L}^{3}}{6 J_{2}^{2}}+\frac{a_{L}^{3}}{6 J_{1}^{2}}-\frac{a_{s}^{3}}{6 J_{3}^{2}}-\frac{a_{s}^{2} t_{4}}{2 J_{3}} .
\end{aligned}
$$


Then, we can get two solutions for $t_{6}$, but it is easy to proove that "+" does not satisfy the condition. Only " - " is saved, as follows.

$$
\begin{aligned}
t_{6}= & \left(\frac{a_{s}}{2 J_{3}}+\frac{a_{L}^{2}}{2 J_{2} a_{s}}-\frac{a_{L}^{2}}{2 J_{1} a_{s}}+\frac{a_{L} t_{2}}{a_{s}}+t_{4}\right) \\
& -\sqrt{\frac{3 a_{L} t_{2}^{2}}{a_{s}}-\frac{6 a_{L} t_{2} t_{4}}{a_{s}}+\frac{3 a_{L}^{2} t_{2}}{J_{2} a_{s}}-\frac{3 a_{L} t_{2}}{J_{3}}+\frac{3 a_{L}^{2} t_{4}}{J_{1} a_{s}}-\frac{3 a_{L}^{2} t_{4}}{J_{2} a_{s}}+\frac{a_{L}^{3}}{J_{2}^{2} a_{s}}-\frac{a_{L}^{3}}{J_{1}^{2} a_{s}}-\frac{a_{L}^{2}}{2 J_{2} J_{3}}+\frac{3 a_{L}^{2}}{2 J_{1} J_{3}}+\frac{a_{s}^{2}}{4 J_{3}^{2}}-\frac{3 a_{L}^{4}}{4 J_{2}^{2} a_{s}^{2}}+\frac{3 a_{L}^{4}}{2 J_{1} J_{2} a_{s}^{2}}-\frac{3 a_{L}^{3} t_{2}}{J_{2} a_{s}^{2}}-\frac{3 a_{L}^{4}}{4 J_{1}^{2} a_{s}^{2}}+\frac{3 a_{L}^{3} t_{2}}{J_{1} a_{s}^{2}}-\frac{3 a_{L}^{2} t_{2}^{2}}{a_{s}^{2}}+\frac{6 S_{\max }}{a_{s}} .}
\end{aligned}
$$

Moreover, $t_{6}$ in (11) satisfied the condition of (5).

3.3. Input Shaping Controller. During dual-stage exchanging process, we pay attention on the communal motor, then all the other kind of forces can be regarded as disturbances. Moreover, the communal motor is a light damping second-order system, with the residual vibration as its typical problem. Input shaping technique can solve this problem based on the information of the closed-loop system, and the demonstration is given as follows. A second-order system's closedloop transfer function usually can be described as follows:

$$
\Phi(s)=\frac{\omega_{n}^{2}}{s^{2}+2 \xi \omega_{n} s+\omega_{n}^{2}},
$$

where $\omega_{n}$ is the damping-free frequency and $\xi$ is the damping ratio. Then, the impulse response of the system at time $t\left(t \geqq t_{i}\right)$ can be written as

$$
\begin{aligned}
y(t) & =\frac{A_{i}}{\sqrt{1-\xi^{2}}} e^{-\xi \omega_{n}\left(t-T_{i}\right)} \sin \left[\omega_{d}\left(t-T_{i}\right)\right], \\
\omega_{d} & =\omega_{n} \sqrt{1-\xi^{2}}
\end{aligned}
$$

where $A_{i}$ is the amplitude, $T_{\mathrm{i}}$ is the starting time of impulse, and $\omega_{\mathrm{d}}$ is the system damping frequency.

If the input is $C(s)=\sum_{i=1}^{n} A_{i} e^{-T_{i} s}$, the response of the system can be expressed as

$$
y(t)=\sum_{i=1}^{n} \frac{A_{i}}{\sqrt{1-\xi^{2}}} e^{-\xi \omega_{n}\left(t-T_{i}\right)} \sin \left[\omega_{n} \sqrt{1-\xi^{2}}\left(t-T_{i}\right)\right],
$$

Videlicet,

$$
y(t)=M \sin \left(\omega_{\mathrm{d}} t+\beta\right),
$$

where

$$
B_{i}=\left(A_{i} \omega_{n} / \sqrt{1-\xi^{2}}\right) e^{-\xi \omega_{n}\left(t-T_{i}\right)},
$$

$M=$

$\sqrt{\left(\sum_{i=1}^{n} B_{i} \cos \phi_{i}\right)^{2}+\left(\sum_{i=1}^{n} B_{i} \sin \phi_{i}\right)^{2}}, \beta=\arctan \left(\sum_{i=1}^{n}\left(B_{i} \cos \right.\right.$ $\left.\left.\phi_{i} / B_{i} \sin \phi_{i}\right)\right)$, and $\phi_{i}=\omega_{d} T_{i}$.

Thus, the residual vibration $V\left(\omega_{\mathrm{n}}, \xi\right)$ can be defined as follows:

$$
V\left(\omega_{n}, \xi\right)=e^{-\xi \omega_{n} T_{n}} M
$$

Subsequently, to achieve residual vibration $V\left(\omega_{n}, \xi\right)=0$, the two pulse zero vibration shaper $(\mathrm{ZV})$ is adopted. By shaping the original reference input, the value of $M$ turns to be zero; thus, no residual vibration exists. In general, defining $T_{1}=0$ could avoid the response delay. At the same time, it is required that the sum of the impulse amplitudes is unity, which ensures that the rigid body actual motion is the same as the unshaped command. Thus, the parameters are given by

$$
\begin{aligned}
& T_{1}=0, \\
& A_{1}=\frac{1}{1+K}, \\
& T_{2}=\frac{\pi}{\omega_{\mathrm{d}}}, \\
& A_{2}=\frac{K}{1+K},
\end{aligned}
$$

where $K=e^{-\left(\xi \pi / \sqrt{1-\xi^{2}}\right)}$.

3.4. AFS-IS Method. As Figure 3 shows, the motion profile named AFS-IS is generated by all free S-curve and input shaper. AFS-IS inherits the advantages of both AFS method and input shaping technology, bringing out the best in each other. For example, AFS method could tune the constraints, for example, distance, force, and impact of motors, while the input shaper cannot do so. Thus, AFS-IS get better performance in constrained motors than input shaping technique. And AFS method can suppress residual vibration in a certain extent, but which cannot be fully eliminated in theory. However, input shaping technique can totally suppress the residual vibration theoretically. In practice, AFS-IS's performance in the residual vibration dispelling is better than both AFS method and input shaping technique. AFS-IS method can be expressed as

$R_{\mathrm{MP}}=\mathrm{IS} \cdot A F S=\sum_{i=1}^{N_{k}} A_{k} \delta\left(t-T_{i}\right) \cdot S\left(J_{1}, a_{L}, t_{2}, J_{2}, t_{4}, J_{3}, a_{S}\right)$,

where $\delta(t)$ is the impulse function at time $t$. 


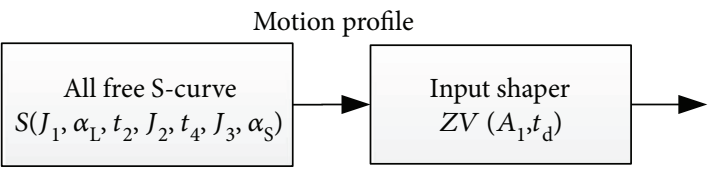

FIGURE 3: Motion profile generated by AFS-IS.

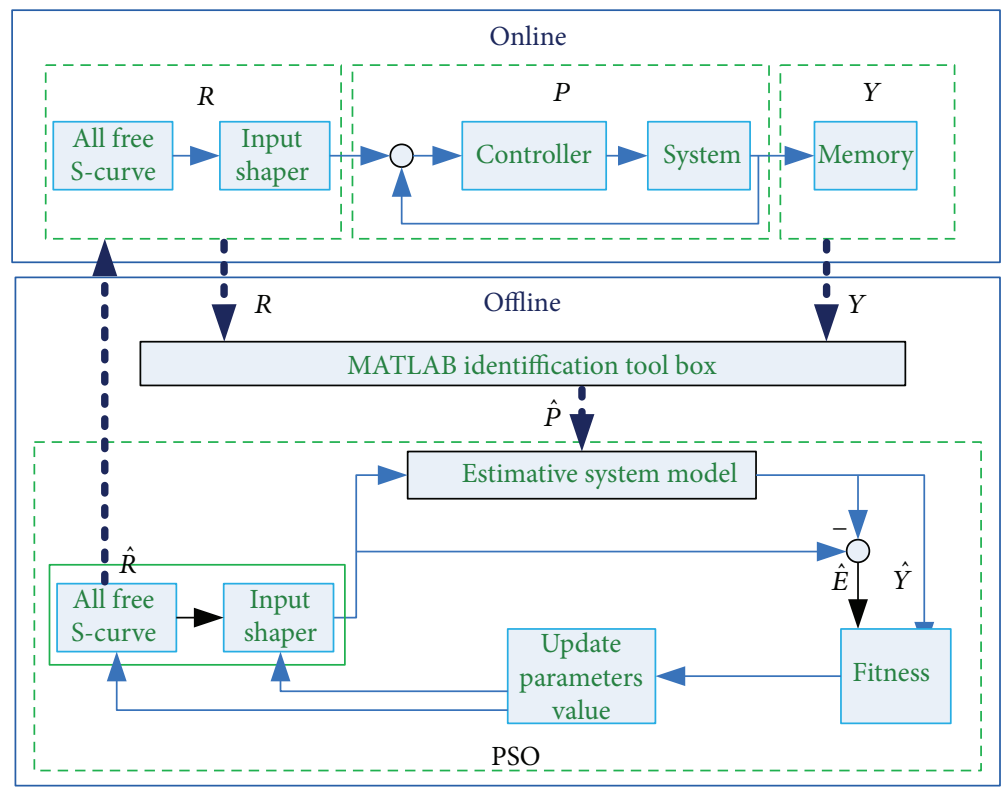

FIGURE 4: Online-offline optimize control system block diagram.

\section{Online/Offline Optimizing Method for Parameters of AFS-IS}

AFS-IS method brings into eight free variables, and it is hard to give analytic solutions to all nine parameters based on the information of the physical system. The optimization method is an alternative solution to get a suitable value of these parameters at the same time. However, there are two objectives, settling time and overshoot, so this paper introduces a common method that uses instrumental variables $\omega_{1}$ and $\omega_{2}$ as weight factors to convert dual-objective problem into single objective problem, which can be served as the objective function of the particle swarm optimization (PSO) algorithm. However, using optimization algorithm to optimize AFS-IS method's parameters online could cause a serious system shock, which may result in instability of the system during iterations, even damage the motor. Therefore, an online/offline method is introduced, which gets the experimental data for modeling online, whereas optimizes the parameters offline.

4.1. Objective Function and Constraints for PSO. The goal of dual-objective optimization in this paper is to find a vector $\mathbf{X}^{*}=\left[\mathbf{x}_{1}{ }^{*}, \mathbf{x}_{2}{ }^{*}, \ldots, \mathbf{x}_{9}{ }^{*}\right]=\left[J_{1}, a_{L}, t_{2}, J_{2}, t_{4}, J_{3}, a_{S}, A_{1}, T_{2}\right]$ that can minimize the settling time and the overshoot. The objective function is described as follows:

$$
\begin{array}{ll}
\min & \omega_{1} \mathrm{~F}_{1}(X)+\omega_{2} \mathrm{~F}_{2}(X) \\
\text { s.t. } & \omega_{1}+\omega_{2}=1,
\end{array}
$$

where $F_{1}$ and $F_{2}$ represent the settling time and the overshoot objective functions, respectively.

It is worth mentioning that the object function of the settling time in this paper is not limited by the steadystate error in twenty-five percent as usual, but the accumulated error from the reference input reaching its target location at time $T_{\eta}$ to termination time $T_{\text {final }}$. And the object function of the overshoot is calculated by the difference between the maximum value of the output $Y_{\sigma}$ and the target location $S_{\max }$.

$$
\begin{aligned}
& F_{1}(X)=\sum_{T_{\eta}}^{T_{\text {final }}}|R(t)-Y(t)|=\sum_{T_{\eta}}^{T_{\text {final }}}|e(t)|, \\
& F_{2}(X)=Y_{\sigma}-S_{\text {max }} .
\end{aligned}
$$

Thus, the objective function can be expressed as

$$
\begin{aligned}
F(X) & =\omega_{1} F_{1}+\omega_{2} F_{2}=\omega_{1} \sum_{T_{\eta}}^{T_{\text {final }}}|e(t)|+\omega_{2}\left(Y_{\sigma}-S_{\max }\right), \\
\omega_{1}+\omega_{2} & =1 .
\end{aligned}
$$

As mentioned in AFS method, there are other inequality constraints in AFS-IS. In detail, the maximum value of output $Y_{\sigma}$ should be larger than that of the target location $S_{\max }$; the settling time $T_{\mathrm{s}}$ should be limited less than the 


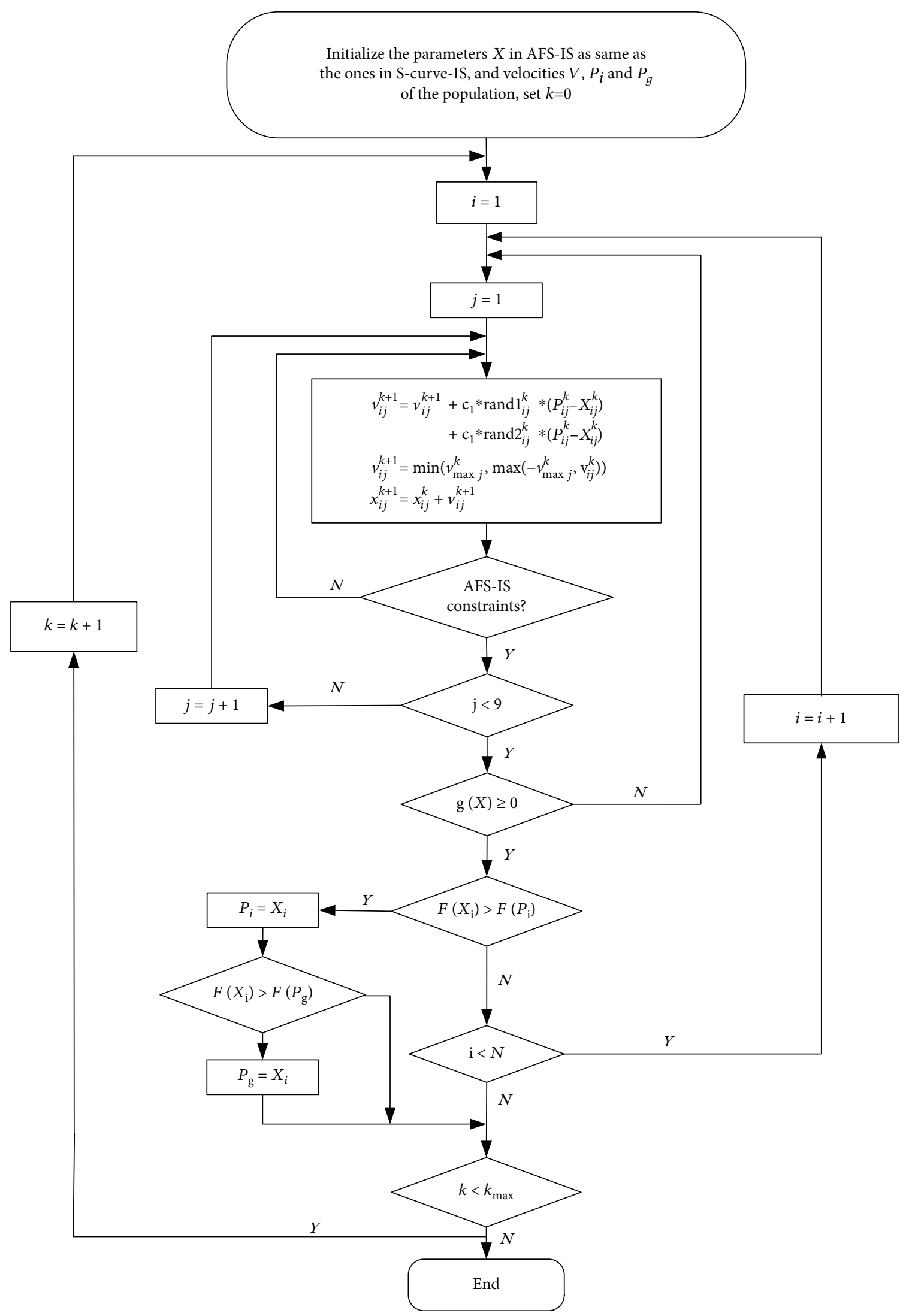

(a) Flow chart of the optimization process

Figure 5: Continued. 

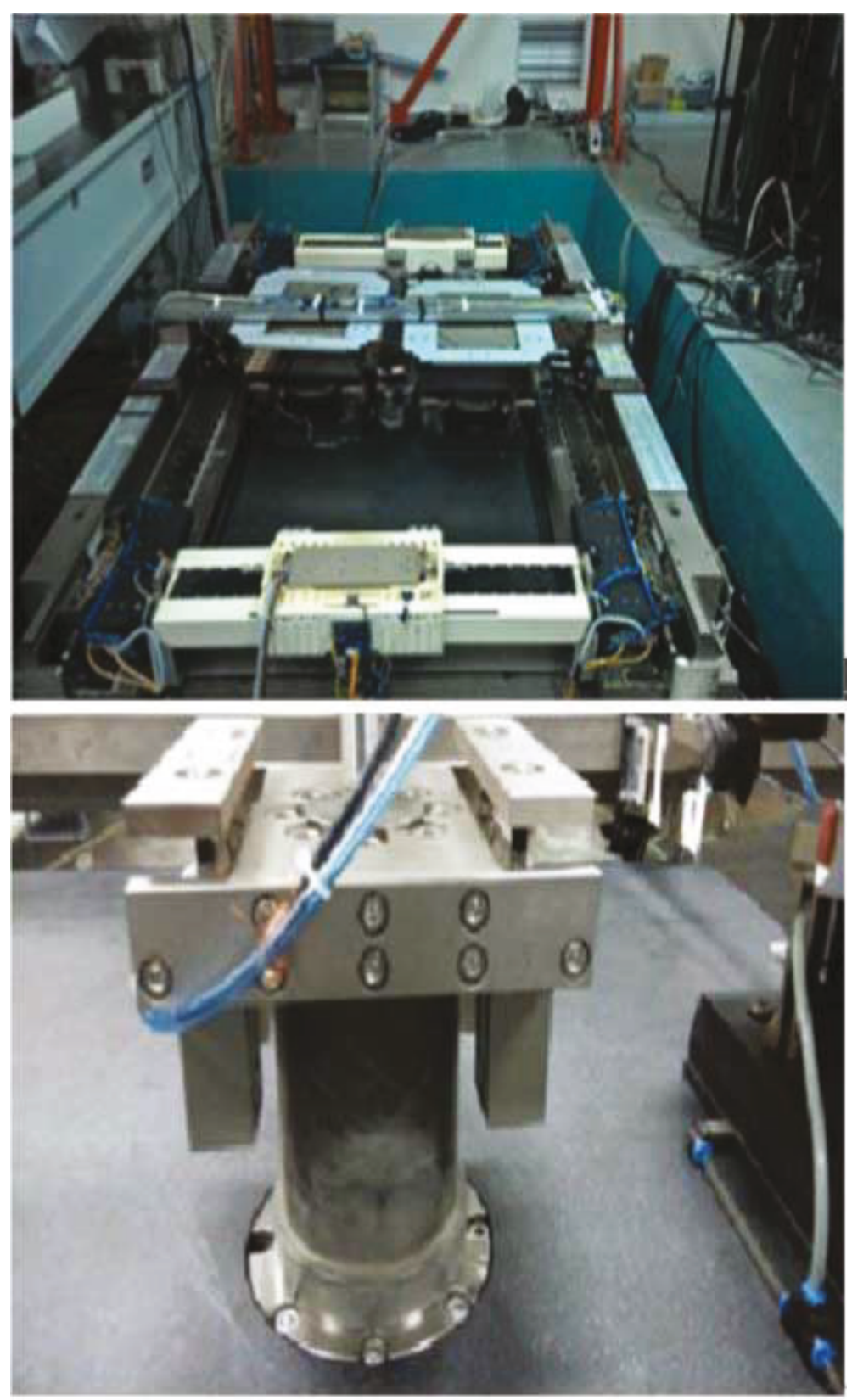

(b) The experimental platform

FIGURE 5: Flow chart of the optimization process and the experimental platform.

maximal allowable movement time $T_{\max }$. Therefore, inequality constraint $g(X)$ can be expressed as

$$
\left.\begin{array}{c}
Y_{\sigma} \geq S_{\max } \\
T_{\max } \geq T_{s} \\
\text { AFS inequality constraints }
\end{array}\right\} \Leftrightarrow g(X) \geq 0 .
$$

Thus, equation constraint $h(X)$ is that in AFS method, and then objective function with equation constraint can be expressed as

AFS equality constraints $\Leftrightarrow h(X)=0$.
As described earlier, the optimization problem is to find a vector $\mathbf{X}^{*}=\left[\mathbf{x}_{1}{ }^{*}, \mathbf{x}_{2}{ }^{*}, \ldots, \mathbf{x}_{8}{ }^{*}\right]$ that satisfies the constraints (21) to minimize the objective vector function.

$$
\begin{gathered}
g(X) \geq 0, \\
h(X)=0, \\
\min \quad F(X) .
\end{gathered}
$$

4.2. An Online-Offline Optimize Motion Profile Generated Method Based on PSO. The diagram of online-offline optimized control system is shown in Figure 4. Firstly, online procedure is using shaped $R$ as the system reference input, 
TABLE 1: MSE between real outputs and identified systems outputs.

\begin{tabular}{lcccccccccc}
\hline $\begin{array}{l}\text { Den } \\
\text { Number }\end{array}$ & 1 & 2 & 3 & 4 & 5 & 6 & 7 & 8 & 9 & 10 \\
\hline 1 & 8.22 & $1.21 e^{-3}$ & $1.65 e^{-3}$ & 2.01 & $9.53 e^{-3}$ & 2.51 & $4.77 e^{-2}$ & $2.92 e^{3}$ & $2.03 e^{-1}$ & $2.74 e^{3}$ \\
2 & - & $6.40 e^{-4}$ & $2.03 e^{-2}$ & $6.41 e^{-4}$ & $4.64 e^{-3}$ & $5.75 e^{-3}$ & 19.5 & $2.79 e^{-3}$ & $1.45 e^{-1}$ & $2.69 e^{3}$ \\
3 & - & - & $3.35 e^{-2}$ & $6.11 e^{-4}$ & $3.21 e^{-4}$ & $4.16 e^{-4}$ & $5.04 e^{-2}$ & 55.6 & $3.37 e^{3}$ & $1.99 e^{3}$ \\
4 & - & - & - & $2.34 e^{-4}$ & $4.05 e^{-4}$ & $4.01 e^{-3}$ & $4.21 e^{3}$ & $3.11 e^{-2}$ & $3.50 e^{3}$ & $3.40 e^{3}$ \\
5 & - & - & - & - & $1.61 e^{-4}$ & $1.46 e^{-4}$ & $4.32 e^{-4}$ & $7.6 e^{-1}$ & $3.91 e^{3}$ & $3.33 e^{3}$ \\
6 & - & - & - & - & - & $\mathbf{1 . 2 4 e ^ { - 4 }}$ & $4.11 e^{3}$ & $6.41 e^{-2}$ & $4.04 e^{3}$ & $1.20 e^{3}$ \\
7 & - & - & - & - & - & - & 3.59 & $2.20 e^{-2}$ & $4.27 e^{3}$ & 2.36 \\
8 & - & - & - & - & - & - & - & $1.50 e^{3}$ & $4.11 e^{3}$ & $1.35 e^{-1}$ \\
9 & - & - & - & - & - & - & - & - & $1.96 e^{-3}$ & $1.42 e^{-2}$ \\
10 & - & - & - & - & - & - & - & - & - & $9.92 e^{-3}$ \\
\hline
\end{tabular}

and after running one time, the actual system output $Y$ is obtained. Secondly, the offline procedure identifies the system model by using MATLAB identification toolbox based on input $R$ and output $Y$, followed by optimizing the parameters in AFS-curve and ZV shaper using PSO algorithm.

The optimizing procedure utilizing PSO is described as follows. Firstly, initialize the parameters $X$ in AFS-IS as same as the ones in conventional S-curve-IS, which means $X_{i}^{k}=\left[J_{1}, a_{L}, t_{2}, J_{2}, t_{4}, J_{3}, a_{S}, A_{1}, T_{2}\right]=\left[J_{1}, a_{L}, t_{2}, J_{1}, t_{4}\right.$, $\left.J_{1}, a_{L}, A_{1}, T_{2}\right]$, and initialize a random velocity $V_{i}^{k}=\left[v_{i 1}^{k}\right.$, $\left.v_{i 2}^{k}, \ldots, v_{i 9}^{k}\right]$, where $i=1,2, \ldots, N$ and $N$ is the total number of particles in the swarm, and $k$ is the iteration index. Secondly, update $X_{i}^{k}$ by (27), then test each particle to judge whether it satisfies the constraints of AFS and IS methods. If no, recreate a particle until it satisfies the condition. If yes, run the simulation with the estimated model that is calculated by the input and output data to acquire the overshoot and the settling time. If $g(X)<0$, recreate a particle until that satisfies $h(X)$ and $g(X)$; otherwise, calculating every particle's fitness value based on the output and the tracking error. Update the best position $X_{i}^{k+1}$ if it is better than the best previous position $P_{i}^{k}$. Finally, update $X_{i}^{k+1}$ if it is better than the best position $P_{g}^{k}$ found by the whole particles, and then begin the next iteration until $k \geq k_{\max }$.

$$
\begin{aligned}
V_{i j}^{(k+1)} & =V_{i j}^{k}+c_{1} r_{1}\left(P_{i j}^{k}-X_{i j}^{k}\right)+c_{2} r_{2}\left(P_{g}^{k}-X_{i j}^{k}\right), \\
v_{i j}^{k+1} & =\min \left(v_{\max j}^{k}, \max \left(-v_{\max j}^{k}, v_{i j}^{k}\right)\right), \\
X_{i j}^{(k+1)} & =X_{i j}^{k}+V_{i j}^{(k+1)},
\end{aligned}
$$

where $c_{1}$ and $c_{2}$ are acceleration coefficients. $r_{1}$ and $r_{2}$ are random values from 0 to 1 .

4.3. Experimental Result. In this section, the proposed AFS-IS method is validated through an experiment on the rotary electric motor shown in Figure 5. The rotary electric motor is driven by proportional-integral-derivative control with a rotary transformer as the feedback signal. To fulfill the prescribed motion profile, a motion control board is used to generate driving signals with a sampling period of $2 \mathrm{~ms}$. To demonstrate that the orders of identified system's numerator and denominator selected are the best one, Table 1 gives the mean square error (MSE) between real outputs and identified system's outputs under different numerator and denominator orders. It can be observed that the system with 6 zeros and 6 poles achieves the least MSE $1.24^{*} 10^{-4}$. Figure 6 shows system actual outputs in the positive and negative direction with 30 degrees and 25 degrees, respectively, compared with the simulation outputs of the identified 6-order system.

To verify the effectiveness of the proposed AFS method, an experiment comparing the AFS method and the conventional S-curve is established. Two constraints for tracking trajectory should be noted. One is distance limitation set to be 30 degrees on the positive direction, and the other is limiting 3 seconds as the longest time for stages to reach at 30 degrees. Meanwhile, two kinds of conventional S-curve as the tracking trajectory are introduced to compare with the AFS method. One is S-S-curve set up with the large maximum jerk, acceleration, and velocity, so it is fast to reach at the object position. On the opposite side, the other is named as L-S-curve with the small maximum jerk, acceleration, and velocity, which is designed to reach at 30 degrees in 3 seconds. The results of the two comparisons are shown in Figures 7(a) and 7(b), respectively, from which it can be observed that the optimized AFS-curve excites less residual vibration. The settling time is reduced by $41 \%$, which is $1 \mathrm{~s}$ with a tolerance, that is, \pm 0.3 degree and overshoot is reduced by $17.4 \%$.

To demonstrate that an additional input shaper can improve the performance of the reference trajectory, the system outputs of AFS and AFS-IS are compared in Figure 8(a), while the system outputs of S-curve with input shaper as the reference trajectory and AFS-IS are compared in Figure 8(b). As Figure 8(a) shows, the input shaper reduces the settling time by $77.2 \%$ and the overshoot by $27.65 \%$. Figure $8(\mathrm{~b})$ demonstrates that, although the S-curve with an input shaper as the reference input gets better system output than a pure S-curve as the reference input, the system output has a 


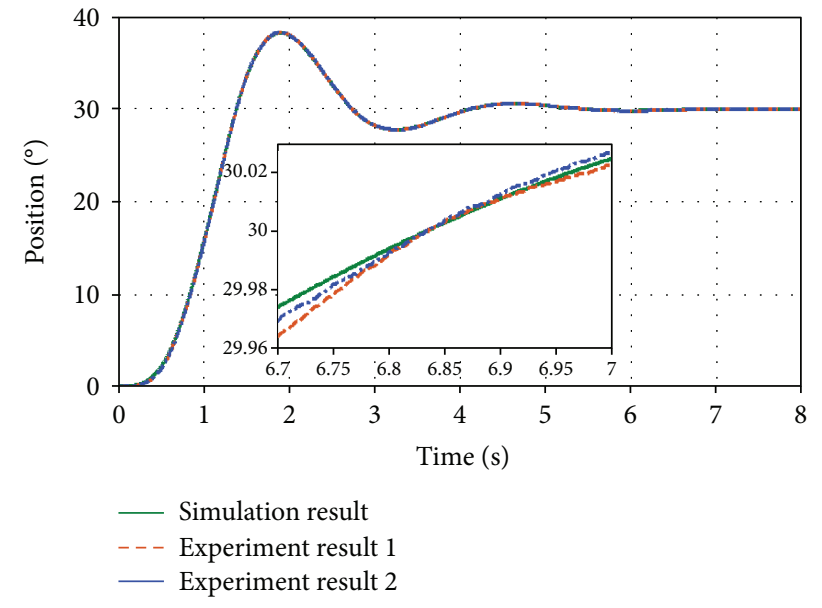

(a) Movement towards the positive direction by $30^{\circ}$

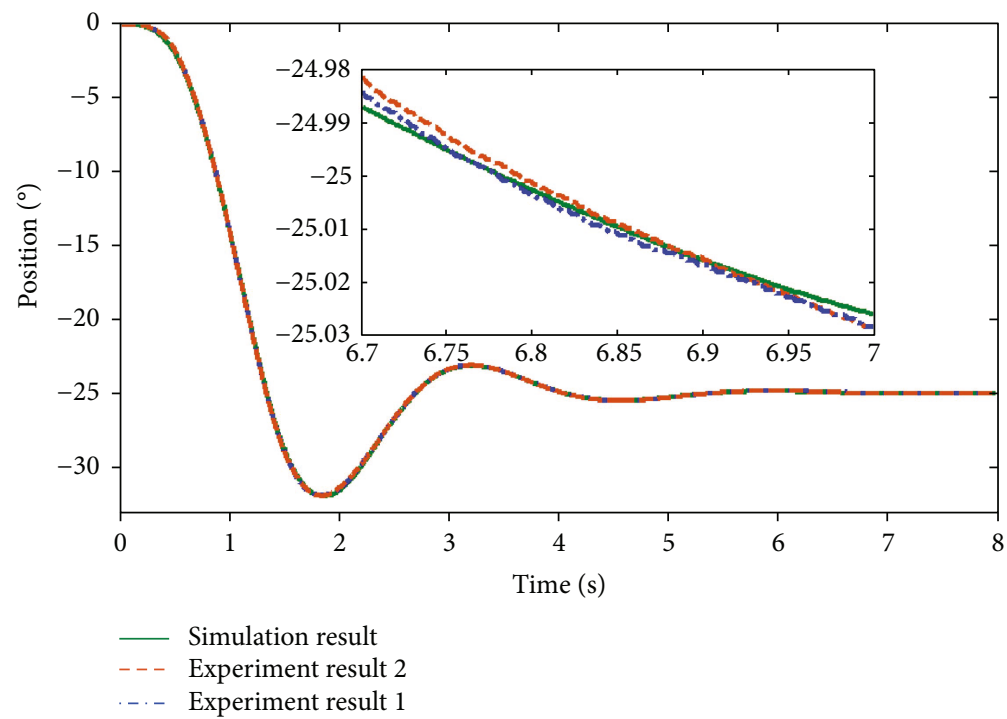

(b) Movement towards the negative direction by $25^{\circ}$

Figure 6: Identified 6-order system simulation outputs and system actual outputs.

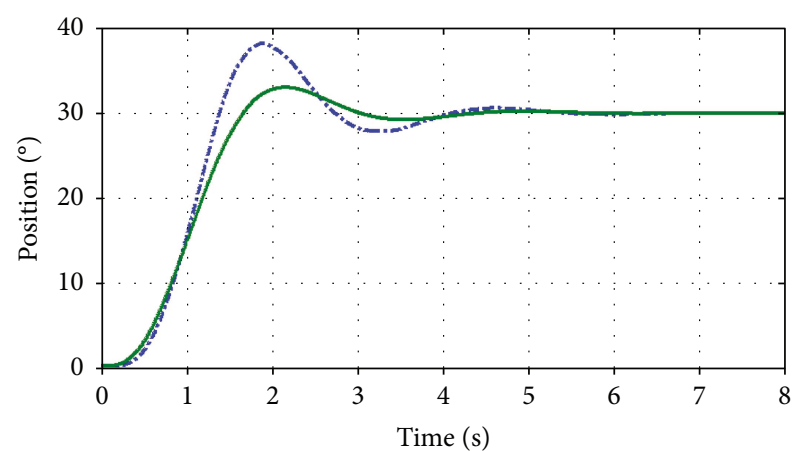

-...- System output with S-S-curve as reference input System output with AFS as refernce input

(a) Comparison between S-S-curve and AFS as reference input

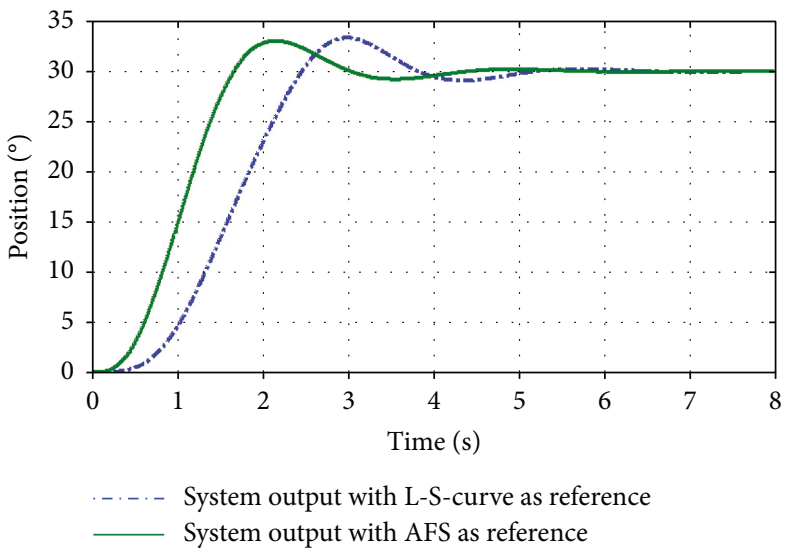

(b) Comparison between L-S-curve and AFS as reference input

Figure 7: Conventional S-curve and AFS-curve as the motion profile. 


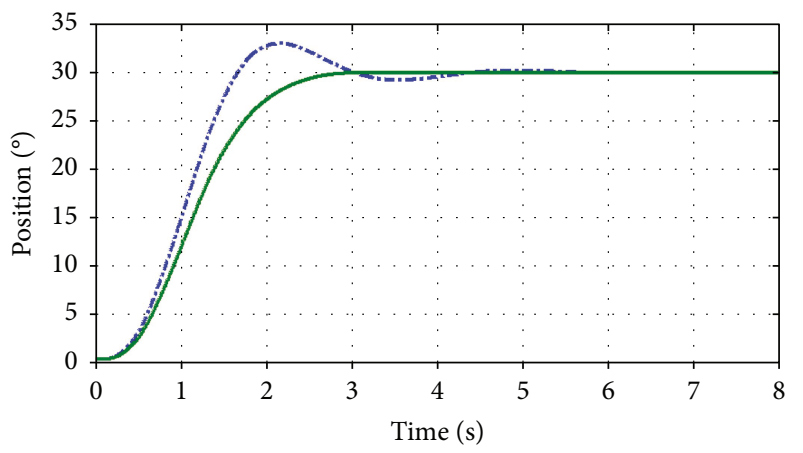

System output with AFS as reference input System output with AFS-IS as reference input

(a) Comparison between AFS and AFS-IS as reference input

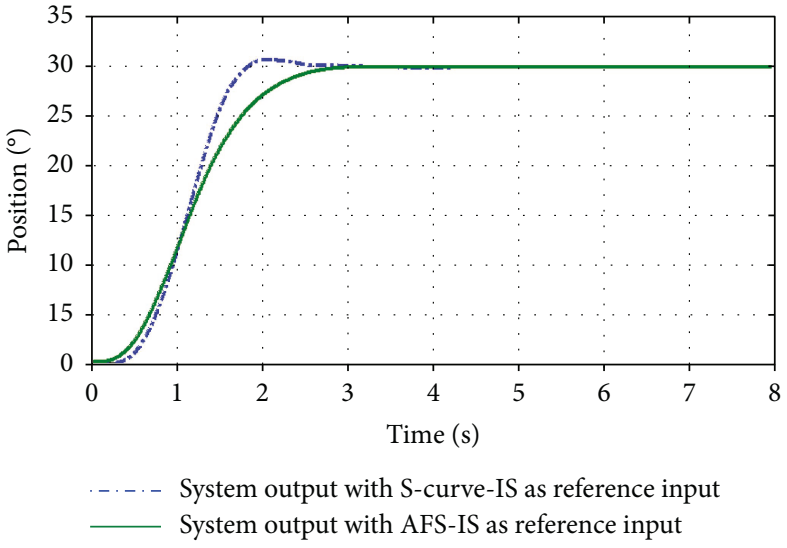

(b) Comparison between S-curve-IS and AFS-IS as reference input

FIgURE 8: Input shaper combined with S-curve and AFS-curve as the motion profile.

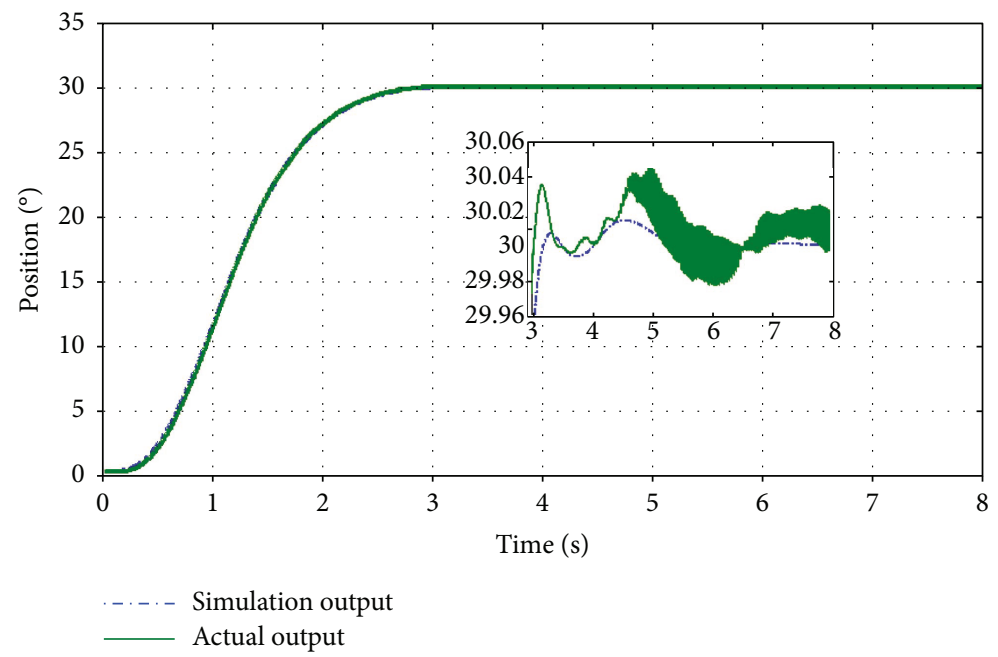

(a) System simulation output and actual output

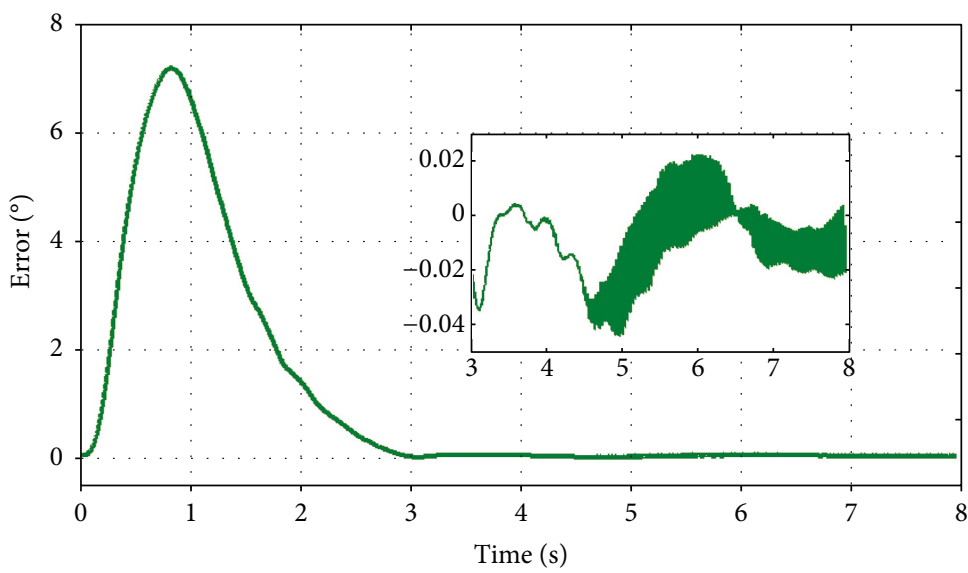

(b) System actual tracking error

FIGURE 9: Actual system output and the positioning error.

$2.45 \%$ overshoot and $1.6 \%$ settling time increment on the system output compared with AFS-IS.

Finally, the online-offline method is verified in the dualstage system. AFS-IS is taken as the motion profile for both simulation system and actual system, and the outputs of two systems are compared in Figure 9(a). With the time changing from 3 seconds to 8 seconds, the actual output is a little larger than the simulation output, and the difference 
between them is within a certain range of 0.035 degrees. Moreover, the difference is caused by the unmodeled dynamics and random disturbances. Figure 9(b) shows the error between the actual output and the AFS-curve. It could be observed that the error changes from 0 to 7 degrees before the 3 rd second, but still in a limitation of 0.05 degrees from the 3 rd second to the 8 th second. For the point to point control, we focus on the error after the 3rd second; thus, the overshoot and the settling time of AFS-IS are significantly reduced compared with the conventional S-curve.

\section{Conclusion}

An all free S-curve combined with a zero-vibration shaper has been proposed to suppress the residual vibration in this paper. The AFS method requires no parameter symmetry during the acceleration and deceleration period, which is more flexible for a better reference trajectory. Furthermore, combined with the input shaping technology, AFS method could achieve a better performance by reducing the overshoot and the residual vibration more effectively. The online-offline method can further enhance the suppression of the residual vibration and avoid the system shock during optimization. The effectiveness of the proposed unified framework is validated on a dual-stage lithographic system. The zero overshoot and the short settling time is achieved by the proposed approach.

\section{Conflicts of Interest}

The authors declare that they have no conflicts of interest.

\section{Acknowledgments}

This work was supported by the State Key Program of National Natural Science of China under Grant 51537002, Chinese National Science Foundation under Grant 51405097, National Science and Technology Major Project under Grant 2017ZX02101007-001, and Scientific Research Foundation for Returned Scholars, Heilongjiang, LC2018022.

\section{References}

[1] F. Song, Y. Wang, X. Chen, and P. He, "Research on input shaping algorithm for rapid positioning of ultra-precision dual-stage," in Proceedings SPIE, Nanoengineering: Fabrication, Properties, Optics, and Devices XII, vol. 95560P, San Diego, CA, USA, August 2015.

[2] B. G. Sluijk, T. Castenmiller, R. du Croo de Jongh et al., "Performance results of a new generation of 300-mm lithography systems," in Proceedings SPIE, Optical Microlithography XIV, vol. 4346, pp. 544-557, Santa Clara, CA, USA, September 2001.

[3] C. Lewin, "Motion control gets gradually better," Machine Design, vol. 66, no. 21, pp. 90-94, 1994.

[4] J. R. García Martínez, J. Rodríguez Reséndiz, M. Á. Martínez Prado, and E. E. Cruz Miguel, "Assessment of jerk performance s-curve and trapezoidal velocity profiles," in 2017 XIII International Engineering Congress (CONIIN), pp. 1-7, Santiago de Queretaro, Mexico, May 2017.
[5] K. H. Rew and K. S. Kim, "Using asymmetric S-curve profile for fast and vibrationless motion," in 2007 International Conference on Control, Automation and Systems, pp. 500-504, Seoul, South Korea, October 2007.

[6] H. Chang-Wan, K. H. Rew, and K. S. Kim, "A complete solution to asymmetric S-curve motion profile: theory \& experiments," in 2008 International Conference on Control, Automation and Systems, pp. 2845-2849, Seoul, South Korea, October 2008.

[7] P. H. Meckl and P. B. Arestides, "Optimized S-curve motion profiles for minimum residual vibration," in Proceedings of the 1998 American Control Conference. ACC (IEEE Cat. No.98CH36207), pp. 2627-2631, Philadelphia, PA, USA, June 1998.

[8] K. D. Nguyen, I.-M. Chen, and T.-C. Ng, "Planning algorithms for S-curve trajectories," in 2007 IEEE/ASME international conference on advanced intelligent mechatronics, pp. 1-6, Zurich, Switzerland, September 2007.

[9] Q. Zang, J. Huang, and Z. Liang, "Slosh suppression for infinite modes in a moving liquid container," IEEE/ASME Transactions on Mechatronics, vol. 20, no. 1, pp. 217-225, 2015.

[10] C. S. Chen and A. C. Lee, "Design of acceleration/deceleration profiles in motion control based on digital FIR filters," International Journal of Machine Tools and Manufacture, vol. 38, no. 7, pp. 799-825, 1998.

[11] T. N. Chang, B. Cheng, and P. Sriwilaijaroen, "Motion control firmware for high-speed robotic systems," IEEE Transactions on Industrial Electronics, vol. 53, no. 5, pp. 1713-1722, 2010.

[12] T. C. Lu, S. L. Chen, and E. C. Y. Yang, "Near time-optimal S-curve velocity planning for multiple line segments under axis constraints," IEEE Transactions on Industrial Electronics, vol. 65, no. 12, pp. 9582-9592, 2018.

[13] R. Eloundou and W. Singhose, "Justification for using stepfunction reference commands: comparison to S-curves," in 2nd IFAC Conference on Mechatronic Systems, Berkeley, CA, USA, 2002.

[14] Q. Zang and J. Huang, "Dynamics and control of threedimensional slosh in a moving rectangular liquid container undergoing planar excitations," IEEE Transactions on Industrial Electronics, vol. 62, no. 4, pp. 2309-2318, 2015.

[15] J. Vaughan, J. Yoo, N. Knight, and W. Singhose, "Multi-input shaping control for multi-hoist cranes," in 2013 American Control Conference, pp. 3449-3454, Washington, DC, USA, June 2013.

[16] O. J. M. Smith, "Posicast control of damped oscillatory systems," Proceedings of the IRE, vol. 45, no. 9, pp. 1249-1255, 1957.

[17] N. C. Singer and W. C. Seering, "Preshaping command inputs to reduce system vibration," Journal of Dynamic Systems, Measurement, and Control, vol. 112, no. 1, pp. 76-82, 1990.

[18] W. E. Singhose, W. Seering, and N. C. Singer, "Residual vibration reduction using vector diagrams to generate shaped inputs," Journal of Mechanical Design, vol. 116, no. 2, pp. 654-659, 1994.

[19] T. Vyhlídal, M. Hromčík, V. Kučera, and M. Anderle, "On feedback architectures with zero-vibration signal shapers," IEEE Transactions on Automatic Control, vol. 61, no. 8, pp. 2049-2064, 2016.

[20] Z. Yang, K. Li, Q. Niu, and Y. Xue, “A novel parallel-series hybrid meta-heuristic method for solving a hybrid unit 
commitment problem," Knowledge-Based Systems, vol. 134, pp. 13-30, 2017.

[21] Z. Yang, K. Li, and A. Foley, "Computational scheduling methods for integrating plug-in electric vehicles in the power system: a review," Renewable and Sustainable Energy Reviews, vol. 51, pp. 396-416, 2015.

[22] Z. Yang, K. Li, Q. Niu, and Y. Xue, “A comprehensive study of economic unit commitment of power systems integrating various renewable generations and plug-in electric vehicles," Energy Conversion and Management, vol. 132, pp. 460-481, 2017.

[23] Z. Chen, Y. Liu, Z. Fu, S. Song, and J. Tan, "Calibration of the hall measurement system for a 6-DOF precision stage using self-adaptive hybrid TLBO," Sensors, vol. 16, no. 6, p. 872, 2016.

[24] F. van den Bergh and A. P. Engelbrecht, "A study of particle swarm optimization particle trajectories," Information Sciences, vol. 176, no. 8, pp. 937-971, 2006.

[25] Y. Zhang, D. W. Gong, and J. Cheng, "Multi-objective particle swarm optimization approach for cost-based feature selection in classification," IEEE/ACM Transactions on Computational Biology and Bioinformatics, vol. 14, no. 1, pp. 64-75, 2017.

[26] C. Ligang, X. Bo, Y. Jianwu, and L. Zhifeng, “The input shaper parameters based on particle swarm optimization tuning algorithm," Electric Machines and Control, vol. 18, no. 10, pp. 87-94, 2014.

[27] S. Sun and J. Li, "A two-swarm cooperative particle swarms optimization," Swarm and Evolutionary Computation, vol. 15, pp. 1-18, 2014.

[28] J. J. Liang, A. K. Qin, P. N. Suganthan, and S. Baskar, "Comprehensive learning particle swarm optimizer for global optimization of multimodal functions," IEEE Transactions on Evolutionary Computation, vol. 10, no. 3, pp. 281-295, 2006.

[29] K. Yu, X. Wang, and Z. Wang, "Multiple learning particle swarm optimization with space transformation perturbation and its application in ethylene cracking furnace optimization," Knowledge-Based Systems, vol. 96, pp. 156-170, 2016. 


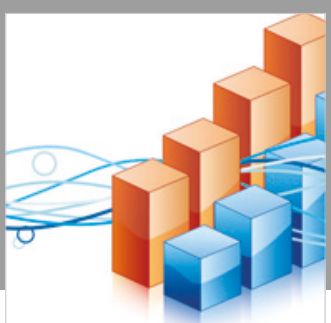

Advances in

Operations Research

\section{-n-m}
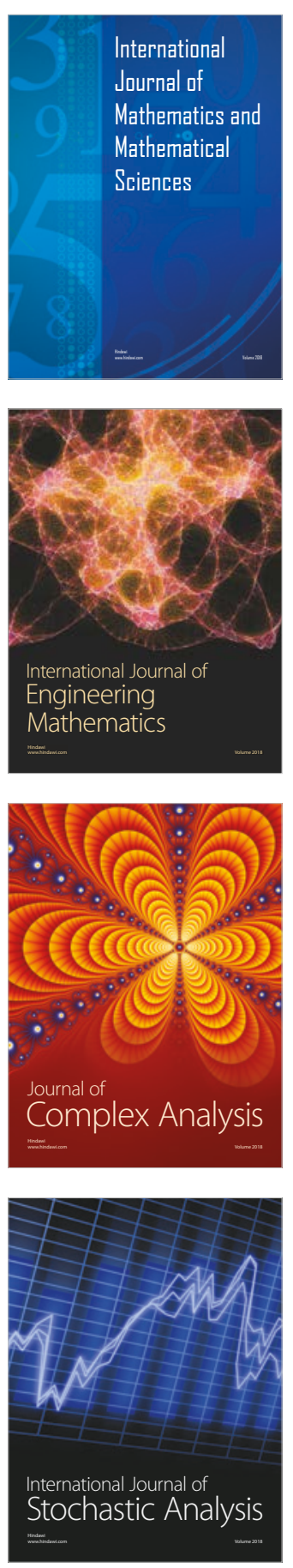
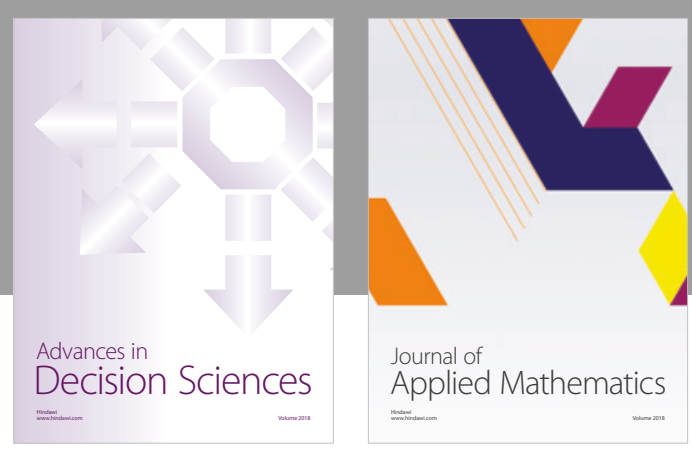

Journal of

Applied Mathematics
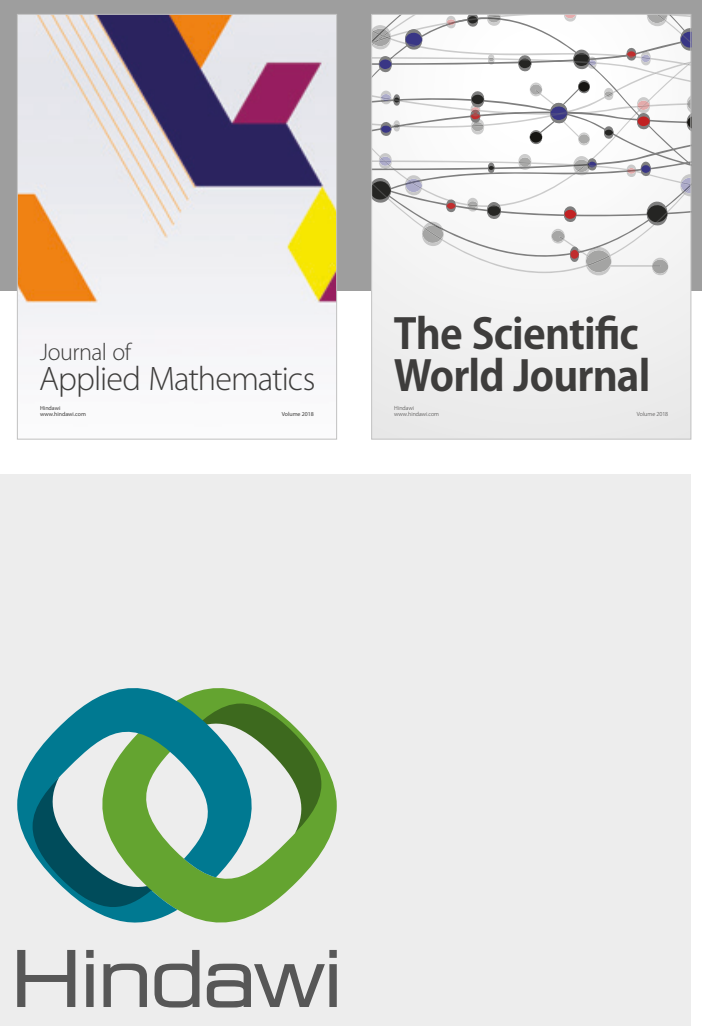

Submit your manuscripts at

www.hindawi.com

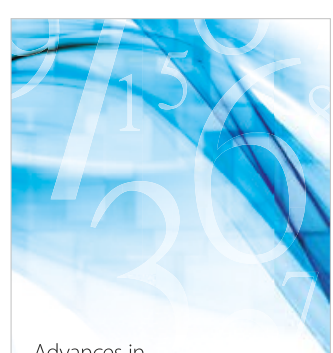

Advances in
Numerical Analysis
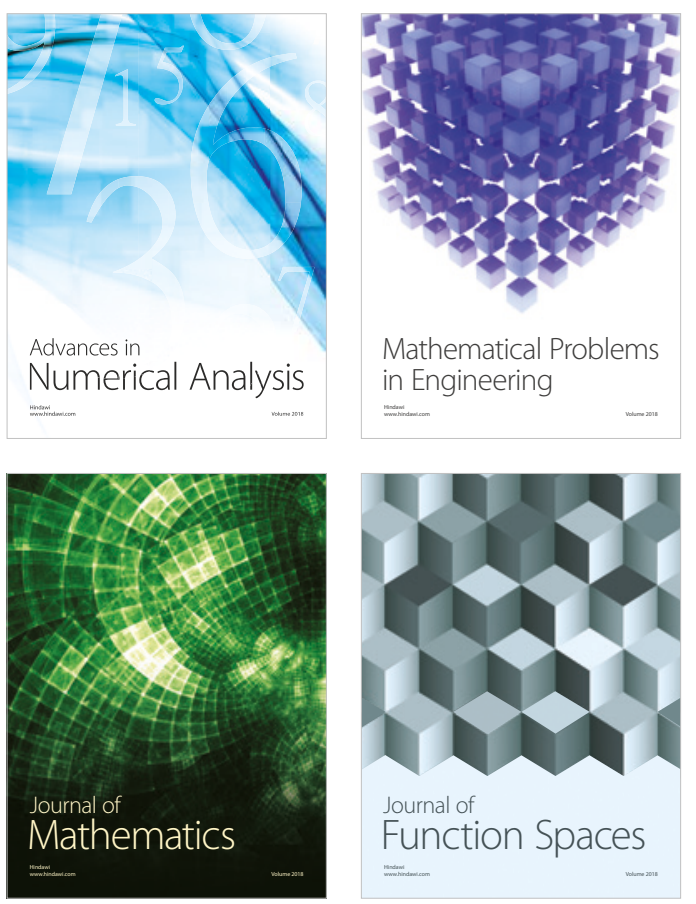

Mathematical Problems in Engineering

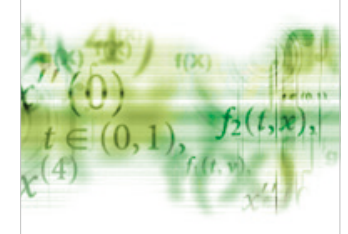

International Journal of

Differential Equations

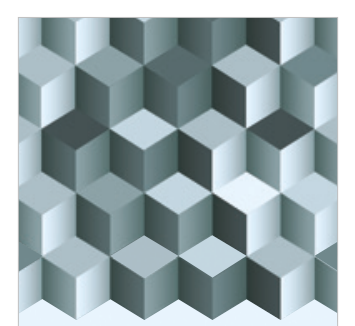

Journal of

Function Spaces
The Scientific

World Journal

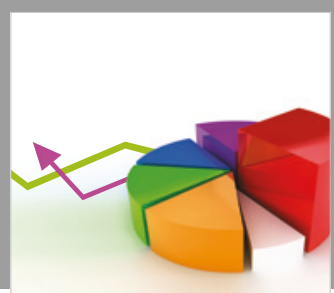

Journal of

Probability and Statistics
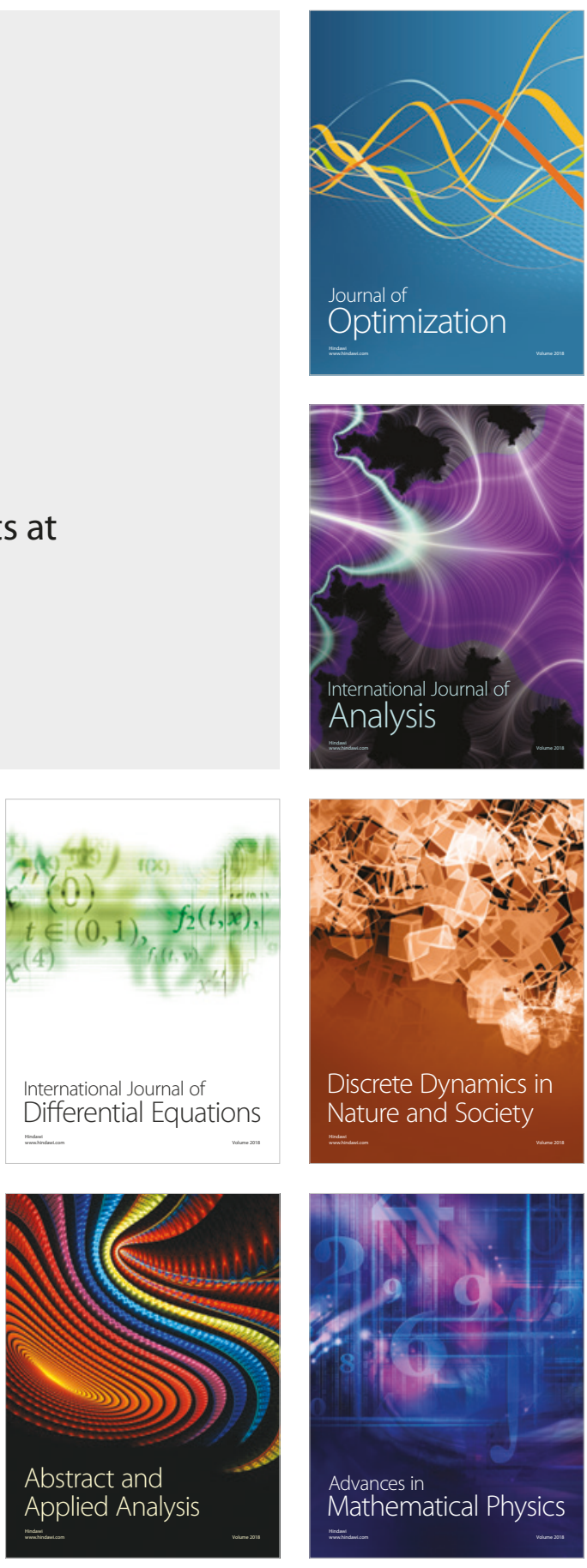